\title{
Ultrasonido Pulmonar en COVID-19
}

\author{
Eugenio Zalaquett ${ }^{*}$, Pascal Lomoro ${ }^{2}$, Anna Natalizi².
}

1. Unidad de Imagenología, CRS Hospital Provincia Cordillera, Servicio de Salud Metropolitano Sur Oriente, Puente Alto, Santiago, Chile.

2. Servicio de Radiología, Ospedale Valduce, Como, Italia.

3. Servicio de Urgencias, Ospedale Valduce, Como, Italia.

\section{Lung Ultrasound in COVID-19}

\section{Resumen:}

La llegada de la pandemia COVID-19 originada por el virus SARS-CoV-2 ha requerido de una rápida respuesta de múltiples especialidades médicas, incluída la Imagenología. El Ultrasonido pulmonar es una técnica que previamente ha demostrado utilidad en otras patologías pulmonares, la cual ofrece alta disponibilidad, bajo costo, ausencia de radiaciones ionizantes y la posibilidad de evaluar al paciente in situ. En algunos de los países afectados más temprana e intensamente por la pandemia, como China e Italia, se ha utilizado como estudio imagenológico complementario para evaluación y seguimiento de los pacientes afectados por esta patología. Para obtener un resultado satisfactorio es necesario seleccionar el equipamiento, protección, desinfección y protocolo adecuados. Los hallazgos ecográficos descritos hasta el momento en la literatura son de carácter inespecífico, pero su combinación y distribución anatómica en un contexto clínico y epidemiológico compatible, los hacen sugerentes de esta enfermedad y permiten evaluar en forma seriada la evolución del cuadro. La literatura disponible es incipiente y es necesaria la generación de más evidencia para cuantificar adecuadamente el aporte de esta modalidad de estudio en los pacientes con COVID-19.

Palabras clave: COVID-19; Pulmonar; Ultrasonido.

\begin{abstract}
:
The arrival of the COVID-19 pandemic originated by the SARS-CoV-2 virus has required a quick response by multiple medical specialties, including Imaging. Lung Ultrasound is an exam that has previously demonstrated its utility in other lung diseases, with high availability, low cost, absence of ionizing radiation and the possibility to have a bed-side assessment. In some of the countries that experienced earlier and more intense effects of the pandemic, as China and Italy, it has been used as an imaging modality for evaluation and follow-up for COVID-19 patients. In order to achieve a satisfactory result, adequate equipment, protection, disinfection and protocol must be chosen. The US findings described so far in the literature are nonspecific, but their combination and anatomical distribution in a compatible clinical and epidemiologic context, made them highly suggestive of this disease and allow for a serial follow-up. The current literature is scarce and more evidence is needed to truly estimate the usefulness of this imaging modality in the evaluation of COVID-19 patients.
\end{abstract}

Keywords: COVID-19; Lung; Ultrasound.

Zalaquett E., et al. Ultrasonido Pulmonar en COVID-19. Rev Chil Radiol 2020; 26(2): 46-51.

${ }^{\star}$ Correspondencia: Eugenio Zalaquett / eugenio.zalaquett@hpcordillera.cl.

Trabajo enviado el 08 de mayo de 2020. Aceptado para publicación el 20 de mayo de 2020. 


\section{Introducción}

El advenimiento de la pandemia COVID-19 generada por un nuevo tipo de Coronavirus denominado SARS-CoV-2, ha traído consigo múltiples desafíos para casi todas las especialidades de la medicina, incluyendo la imagenología. El método de estudio mejor caracterizado en términos globales ha sido la TC. No obstante, diversos centros hospitalarios, principalmente en países que fueron precoz e intensamente afectados por la pandemia como China e Italia, hicieron uso del ultrasonido en el punto de atención (Point-of-care ultrasound, POCUS) como parte de la evaluación imagenológica de los pacientes con COVID-19 ${ }^{(1,2)}$. Previamente, se ha documentado la utilidad del ultrasonido pulmonar en el estudio diversas patologías, tales como el edema pulmonar cardiogénico, el edema pulmonar inflamatorio, la fibrosis pulmonar, las consolidaciones pulmonares, el derrame pleural y el neumotórax ${ }^{(3)}$.

Si bien no es posible realizar el diagnóstico de COVID-19 a través del ultrasonido (4), su uso complementario en el seguimiento de la evolución de los pacientes y su capacidad para orientar a diagnósticos alternativos han sido valorada por los expertos en el contexto de los primeros reportes publicados ${ }^{(5,6)}$.

El ultrasonido presenta múltiples ventajas frente a otros métodos de estudio imagenólogico: ausencia de uso de radiaciones ionizantes, bajo costo comparativo, alta disponibilidad y posibilidad de realizar exámenes dinámicos y en cualquier lugar. En el caso específico del COVID-19, existen algunas ventajas adicionales. En primer lugar, los equipos de ultrasonido, ya sean de bolsillo, portátiles o estacionarios; son más fáciles de limpiar y desinfectar que los equipos de radiografía o de TC, lo que otorga una ventaja considerable en términos de poder llevar a cabo protocolos efectivos de control de la transmisión del virus asociada a atenciones de salud ${ }^{(7)}$. Además, aquellos pacientes que desarrollan la variante más grave de la enfermedad, con neumonitis viral y requerimiento de ventilación mecánica invasiva, se benefician especialmente de contar con una modalidad de estudio que puede ser realizada en la misma Unidad de Tratamiento Intensivo (UTI), en forma reiterada, obviando los riesgos propios del traslado intrahospitalario.

\section{Preparación antes del examen}

Para lograr una adecuada evaluación ecográfica de los pacientes con COVID-19, el proceso debe iniciarse mucho antes de la realización del examen en cuestión. Para lograr un resultado satisfactorio es fundamental seleccionar adecuadamente el tipo de ecógrafo a utilizar, el transductor, los elementos de protección para personal y equipamiento y los métodos de desinfección.

Los equipos ideales para realizar este examen, según lo reportado por algunos de los grupos que han tenido mayor experiencia en este campo, son los ecógrafos de bolsillo. Estos equipos, la mayoría de los cuales están constituidos por un transductor que transmite la imagen a un teléfono o una tableta, permiten una adecuada visualización pulmonar periférica a la vez que poseen mínimas superficies susceptibles de ser contaminadas, además de presentar un costo relativo menor ${ }^{(7)}$. Sin embargo, estos equipos aún ostentan baja penetración y disponibilidad en el medio chileno. Los equipos portátiles son la segunda mejor alternativa, ya que presentan en términos generales una buena capacidad técnica en asociación con un tamaño disminuido y muchos de ellos incorporan el uso de pantallas táctiles para facilitar su operación en un contexto bedside o son resistentes a fluidos. Por último, están los equipos estacionarios tradicionales, los que habitualmente ofrecen una mejor capacidad técnica, pero pueden ser poco prácticos para ser utilizados de manera móvil con pacientes hospitalizados, particularmente en un contexto de cuidados intensivos y ventilación mecánica invasiva. No obstante, sea cual sea el equipo del que se disponga, la recomendación es que sea de uso exclusivo para pacientes COVID-19 o con sospecha de esta patología, de manera tal de evitar la transmisión del virus tanto a pacientes internados por otras causas como al resto del personal sanitario( ${ }^{(8)}$.

En lo que refiere a la selección del transductor, existe una variedad de recomendaciones en la literatura. En términos generales se recomienda el uso de un transductor de baja frecuencia, de 3 a $5 \mathrm{MHz}$, como el característico transductor curvo abdominal, para lograr una penetración de 12 a $18 \mathrm{~cm}$ (dependiendo de las dimensiones corporales del paciente). Los transductores microconvexos ofrecen la ventaja de una huella de menor tamaño que permite mejor visualización intercostal, especialmente en pacientes más pequeños. Sin embargo, son los transductores lineales de alta resolución los que ofrecen una mejor visualización de la línea pleural y de lesiones subpleurales pequeñas ${ }^{(9)}$. La inmensa mayoría de los equipos actuales incluyen de fábrica configuraciones especiales para ecografía pulmonar. Sin embargo, en los equipos más antiguos que no las posean, se recomienda desactivar las características de emisión o post procesamiento que tienen como fin la disminución de la generación de artefactos, tales como el uso de armónicos o de composición espacial, ya que los artefactos son fundamentales para interpretar los hallazgos en este examen en especial ${ }^{(8)}$.

Respecto de los elementos de protección del equipo, se sugiere la utilización de cobertores desechables de plástico transparente para transductores y cables, los que no limitan la visualización ni generan artefactos significativos. En caso de usar equipos estacionarios que se encuentren en la cercanía del paciente, se pueden utilizar también cobertores 
plásticos transparentes que cubran las superficies del equipo, especialmente aquellas que han de ser manipuladas como las perillas o el teclado.

En términos de higienización del equipo se recomienda realizar un proceso de desinfección antes y después de cada examen realizado(10). Respecto de los medios químicos utilizados para llevar a cabo dicha desinfección, es fundamental tener conocimiento de las recomendaciones del fabricante del equipo respecto de las sustancias permitidas para su limpieza. En forma muy general, se sabe que el uso de soluciones que contengan alcohol es deletéreo para la inmensa mayoría de los transductores. La Agencia de Protección Ambiental de EE.UU. (Environmental Protection Agency, EPA) ha publicado un listado con todas las sustancias que son efectivas en contra del SARS-CoV-2 junto al tiempo necesario de exposición al agente químico ${ }^{(11)}$.

\section{Examen}

Existen diversos protocolos de ecografía pulmonar. Sin embargo, los reparos anatómicos que se utilizan para dividir los campos de exploración son comunes a muchos de ellos. A cada lado, el campo anterior se ubica entre la línea paraesternal y la línea axilar anterior. El campo lateral se ubica entre la línea axilar anterior y la línea axilar posterior. El campo posterior se ubica entre la línea axilar posterior y la línea paravertebral. A su vez, cada uno de estos campos se subdivide en superior e inferior en relación, aproximadamente, al tercer espacio intercostal. Esto da un total de 12 campos (6 por cada pulmón) (Figura 1) ${ }^{(6,12)}$. Existen variantes de este protocolo en donde se agregan subdivisiones adicionales o se omiten los campos posteriores por la condición clínica del paciente ${ }^{(8)}$.

En cada uno de estos campos, la visualización se realiza a través de los espacios intercostales, con el eje largo del transductor en situación perpendicular a los arcos costales. Esto genera imágenes en las cuales los contornos costales y sus respectivas sombras acústicas son claramente identificables, alternándose con áreas intercostales que incluyen en forma secuencial piel, tejido celular subcutáneo, plano muscular, línea pleural y parénquima pulmonar periférico. El tejido pulmonar sano, adecuadamente ventilado, genera artefactos de reverberación que nacen desde la línea pleural conocidos como "Líneas A" (Figura 2), debido a la interfaz generada por el aire contenido en condiciones normales en los alvéolos y el parénquima propiamente tal. Estas líneas son de orientación claramente horizontal y discontinua, exhibiendo una distancia igual entre cada línea individual reverberada ${ }^{(13)}$. Además, en un examen normal será posible evidenciar con claridad una delgada y regular línea pleural que se desliza notoriamente en correspondencia con los movimientos ventilatorios del tórax.

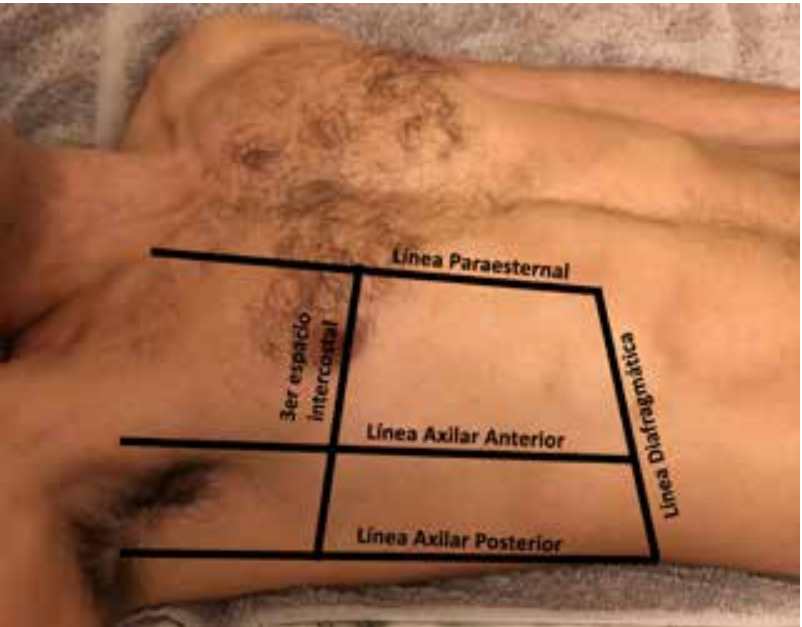

Figura 1: Topografía de los campos a explorar en un protocolo de 12 campos (6 por pulmón). Entre la línea paraesternal y la línea axilar anterior se ubica el campo anterior. Entre la línea axilar anterior y la línea axilar posterior se ubica el campo lateral. Entre la línea axilar posterior y la línea paralumbar (no mostrada) se ubica el campo posterior. $A$ su vez, cada campo se subdivide en superior e inferior.

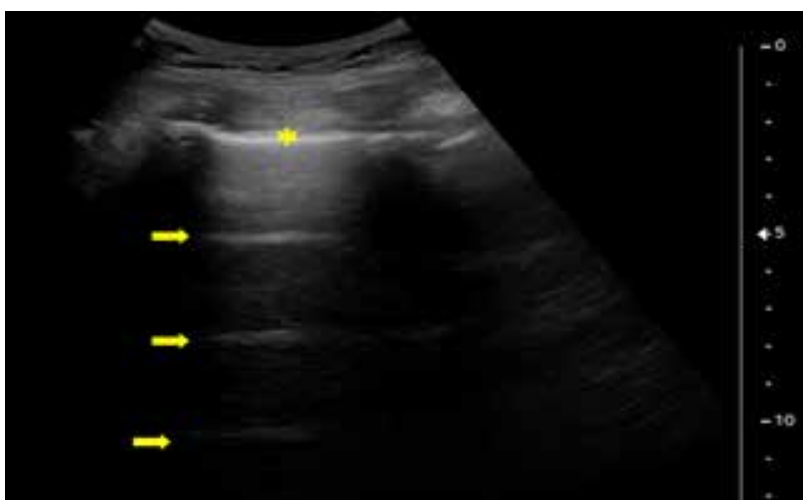

Figura 2: Pulmón normal. Los arcos costales son claramente identificables por su sombra acústica posterior. El espacio intercostal se observa nítidamente la línea pleural (asterisco), la que es hiperecogénica, delgada y lisa. A partir de ella se observan 3 líneas de igual disposición y espesor (flechas), separadas por espacios de igual magnitud entre ellas. Estas constituyen "Líneas $A$ " y corresponden al artefacto de reverberación generado por un parénquima pulmonar subpleural que presenta una proporción conservada entre aire y componentes tisulares.

En ecografía pulmonar no existen hallazgos que sean específicos para COVID-19 ${ }^{(13)}$, pero su asociación y distribución son altamente sugerentes en un contexto clínico compatible, más aún en un contexto epidemiológico como el actual. Se ha descrito que los hallazgos en Ultrasonido tienen una distribución de 
predominio posterior, más especialmente posteroinferior. Por ello, en aquellos pacientes de movilidad reducida se sugiere aprovechar instancias en las cuales son movilizados para tener acceso a los campos posteriores, como por ejemplo en los cambios de posición de los protocolos de prevención de escaras o durante las sesiones de kinesioterapia. Otra consideración importante es la existencia concomitante de áreas con hallazgos positivos y de áreas ecográficamente normales, también llamadas áreas respetadas. La existencia de hallazgos simétricos en todos los campos hará más sugerente un origen cardiogénico antes que etiología inflamatoria-infecciosa, especialmente COVID-19(3).

El principal hallazgo ecográfico corresponde a las denominadas "Líneas B", las que constituyen un artefacto de reverberación que nace, al igual que las líneas A, a partir de la línea pleural (Figura 3). Sin embargo, se diferencian de estas últimas por ser hiperecogénicas, mejor definidas, de disposición más bien vertical y continua, aunque también se desplazan en conjunto con los deslizamientos pleurales ${ }^{(9)}$. Pueden aparecer en forma aislada o confluente, llegando en casos más severos a constituir un "pulmón blanco ecográfico" ("white lung") (Figura 4), el que se correlaciona con el hallazgo de densidad de vidrio esmerilado en tomografía computada ${ }^{(14)}$. Si bien las líneas B constituyen un hallazgo inespecífico que puede verse en múltiples patologías pulmonares en las que existe una pérdida de la proporción habitual entre aire y parénquima pulmonar, la distribución bilateral de predominio posteroinferior con presencia de áreas respetadas, son compatibles con COVID-19 en un contexto clínico pertinente ${ }^{(7,15)}$.

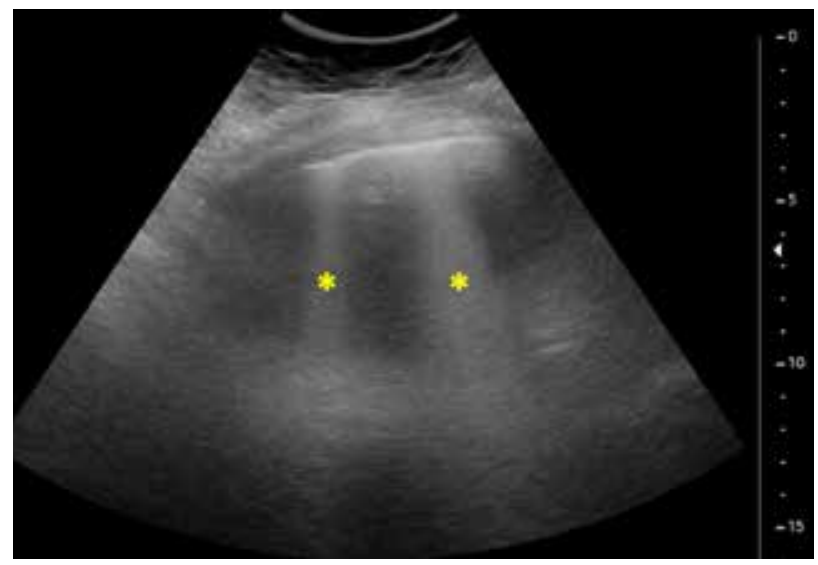

Figura 3: Paciente masculino de 73 años de edad con sintomatología respiratoria y fiebre. RT-PCR de muestra nasofaríngea positiva para COVID-19. A partir de la línea pleural se identifican dos artefactos de reverberación hiperecogénicos (asteriscos), continuos, que llegan hasta el final de la pantalla, los que corresponden a "Líneas B".

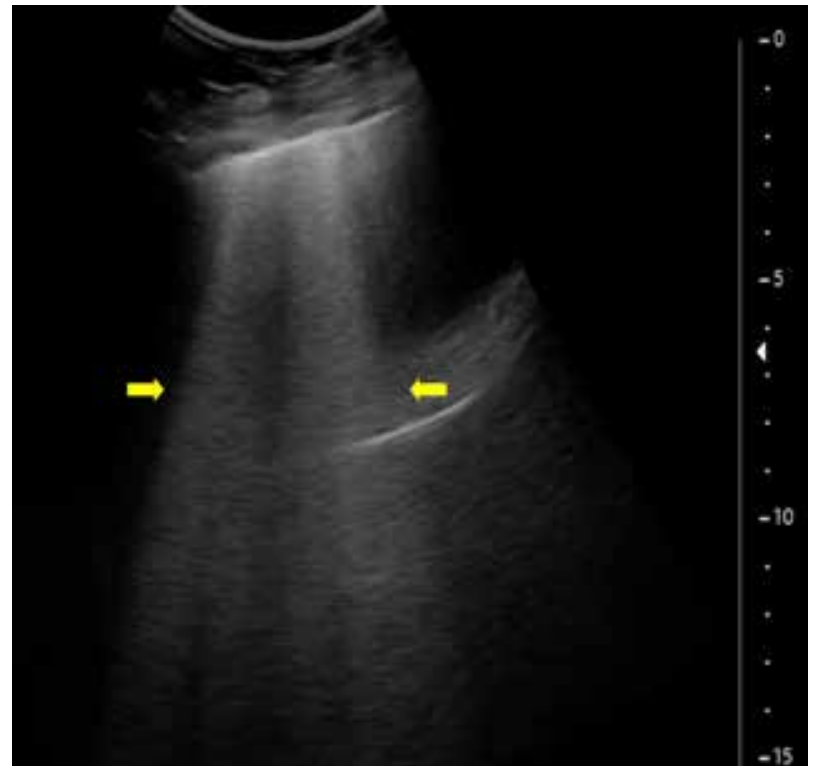

Figura 4: Paciente masculino de 77 años de edad con insuficiencia respiratoria. RT-PCR de muestra nasofaríngea positiva para COVID-19. A partir de la línea pleural se identifican múltiples Líneas $B$ coalescentes (flechas), cuyos límites no pueden identificarse en forma categórica, ocupando la totalidad del espesor del espacio intercostal examinado. Esto es lo que se conoce como "pulmón blanco" (white lung).

El segundo hallazgo asociado a COVID-19 es irregularidad y/o engrosamiento de la línea pleural, especialmente si se alterna con áreas en las cuales ésta se visualiza indemne. La línea pleural habitualmente es continua y de aspecto liso. Cabe destacar que un compromiso difuso de la línea pleural, sin áreas de respeto, es propio de las enfermedades pulmonares intersticiales fibróticas.

Otro hallazgo descrito en COVID-19 es la visualización de consolidaciones subpleurales, las que en casos más tenues pueden ser identificadas a través de la interrupción focal de la línea pleural (Figura 5). Cuando la consolidación es de mayor cuantía, el parénquima pulmonar puede adquirir un aspecto ecográfico similar al parénquima hepático sano (hepatización) (Figura 6) y puede exhibir imágenes hiperecogénicas estáticas o móviles que corresponden al correlato ecográfico del broncograma aéreo descrito en TC. Sin embargo, cuando existe una consolidación única, de mayor tamaño, es más probable una neumonía bacteriana lobar en ausencia de hallazgos adicionales.

La visualización de derrame pleural en cuantía significativa o de tabiques pleurales, orientan en la dirección de una etiología alternativa a COVID-19, ya que son hallazgos infrecuentes en esta patología. No 
obstante, no es raro evidenciar discretos bolsillos de derrame pleural focal en situación inmediatamente adyacente a consolidaciones subpleurales originadas por esta enfermedad.

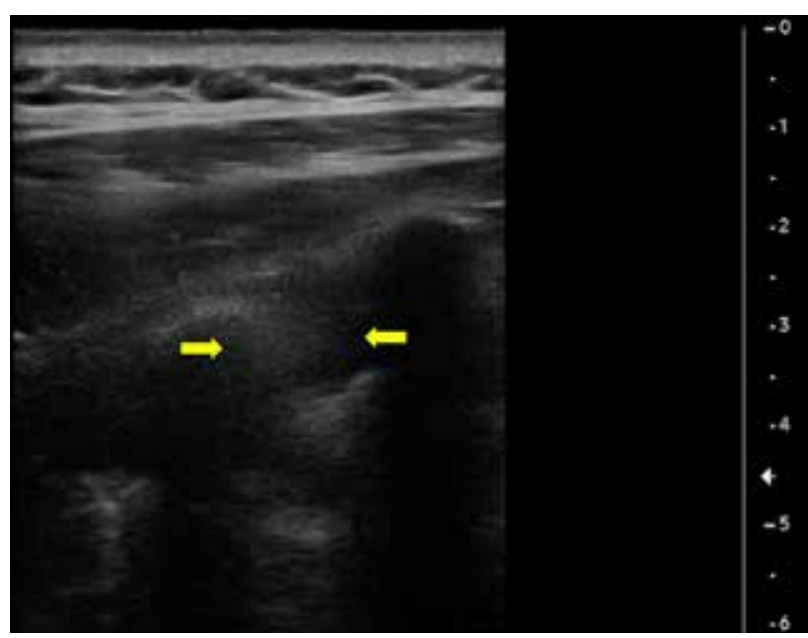

Figura 5: Paciente femenino de 81 años de edad con insuficiencia respiratoria. RT-PCR de muestra nasofaríngea positiva para COVID-19. Se identifica una interrupción de la línea pleural en el contexto de una imagen de ecogenicidad intermedia (flechas) que determina ausencia de artefactos de reverberación a ese nivel, compatible con una condensación subpleural.

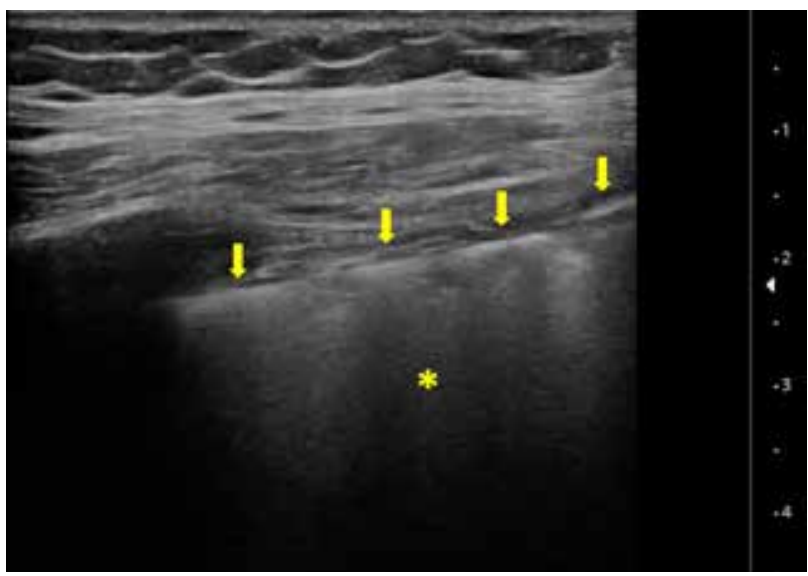

Figura 6: Paciente masculino de 76 años de edad con sintomatología respiratoria. RT-PCR de muestra nasofaríngea positiva para COVID-19. La línea pleural presenta al menos 4 segmentos de espesor aumentado alternados (flechas). Inmediatamente por debajo se observa un patrón ecográfico reminiscente del parénquima hepático (asterisco), con ausencia de artefactos de reverberación. Esto corresponde a una condensación subpleural de mayor cuantía con "hepatización pulmonar".

\section{Conclusión}

El Ultrasonido es una técnica de estudio por imágenes de bajo costo y alta disponibilidad que no utiliza radiaciones ionizantes. En el contexto de la pandemia por COVID-19 ha mostrado utilidad como modalidad complementaria de estudio imagenológico en la atención multidisciplinaria de los pacientes afectados por esta enfermedad, especialmente de aquellos que manifiestan la variante más severa, con requerimiento de cuidados intensivos y ventilación mecánica invasiva.

La asociación de los hallazgos ecográficos descritos, con una distribución bilateral multifocal y en un contexto clínico-epidemiológico compatible, es sugerente de infección por SARS-CoV-2. Sin embargo, aún está pendiente la generación de evidencia médica de mayor calidad que cuantifique de manera razonable su aporte real en los distintos escenarios clínicos.

Agradecimientos: a la Dra. Francesca Maio, Médico Radiólogo del Ospedale Anastasia Guerriero de Marcianise, Italia y al Dr. Luis Vergara, Médico Internista y Especialista en Medicina del Deporte de Clínica QuironSalud del Valles Sabadell, Barcelona, España.

\section{Referencias}

1. Moore S, Gardiner E. Point of care and intensive care lung ultrasound: A reference guide for practitioners during COVID-19 (published online ahead of print, 2020 Apr 17). Radiography (London). 2020; S10788174(20)30057-2.

2. Sikachi R, Agrawal A. Whole body point of care ultrasound for COVID-19: a multi-system approach to a multi-system disease. Anaesthesia. 2020; 2019: 2019-20.

3. Volpicelli G, Elbarbary M, Blaivas M, Lichtenstein DA, Mathis G, Kirkpatrick AW, et al. International evidencebased recommendations for point-of-care lung ultrasound. Intensive Care Med. 2012; 38(4): 577-591.

4. Pierce $\mathrm{CW}$. Clarifying the role of lung ultrasonography in COVID-19 respiratory disease. Cmaj. 2020; 192(16): E436.

5. Volpicelli G, Mussa A, Garofalo G, Cardinale L, CasoliG, Perotto F, et al. Bedside lung ultrasound in the assessment of alveolar-interstitial syndrome. Am J Emerg Med. 2006; 24(6): 689-696.

6. Peng QY, Wang XT, Zhang LN; Chinese Critical Care Ultrasound Study Group (CCUSG). Findings of lung ultrasonography of novel corona virus pneumonia during the 2019-2020 epidemic. Intensive Care Med. 2020; 46(5): 849-850.

7. Ramanathan $\mathrm{K}$, Antognini D, Combes $\mathrm{A}$, Paden $\mathrm{M}$, Zakhary B, Ogino M, et al. Since January 2020 Elsevier has created a COVID-19 resource centre with free information in English and Mandarin on the novel coronavirus COVID- research that is available on the COVID-19 resource centre - including this for unrestricted research re-use a. 2020 January: 19-21. Available from: https://www.ncbi.nlm.nih.gov/pmc/articles/PMC7127800/ pdf/main.pdf

8. Soldati G, Smargiassi A, Inchingolo R, Buonsenso D, 
Perrone T, Briganti DF, et al. Proposal for international standardization of the use of lung ultrasound for COVID-19 patients; a simple, quantitative, reproducible method. J Ultrasound Med. 2020; 1-7.

9. Lee FC. Lung ultrasound-a primary survey of the acutely dyspneic patient. J Intensive Care. 2016; 4(1): 57. Published 2016 Aug 31.

10. Soldati G, Smargiassi A, Inchingolo R, Buonsenso D, Perrone T, Briganti DF, et al. Is there a role for lung ultrasound during the COVID-19 pandemic? J Ultrasound Med. 2020; 1-4.

11. Agency USEP. List N: Disinfectants for Use Against SARSCoV-2. Available from: https://www.epa.gov/pesticideregistration/list-n-disinfectants-use-against-sars-cov-2

12. Yi Huang, Sihan Wang, Yue Liu, et al. A preliminary study on the ultrasonic manifestations of peripulmonary lesions of non-critical novel coronavirus pneumonia (COVID-19); 26 February 2020, PREPRINT (Version 1) available at Research Square. https://doi.org/10.21203/rs.2.24369/ v1+.

13. Vetrugno L, Bove T, Orso D, Barbariol F, Bassi F, Boero $\mathrm{E}$, et al. Our Italian experience using lung ultrasound for identification, grading and serial follow-up of severity of lung involvement for management of patients with COVID-19. Echocardiography. 2020 March; 625-627.

14. Sofia S, Boccatonda A, Montanari M, et al. Thoracic ultrasound and SARS-COVID-19: a pictorial essay. J Ultrasound. 2020; 23(2): 217-221. doi:10.1007/s40477020-00458-7

15. Volpicelli G, Gargani L. Sonographic signs and patterns of COVID-19 pneumonia. Ultrasound J. 2020; 12(1): 22. Published 2020 Apr 21. 